\title{
Heme oxygenase-1 gene induction as an intrinsic regulation against delayed cerebral vasospasm in rats
}

\author{
Hidenori Suzuki, ${ }^{1,2}$ Kenji Kanamaru, ${ }^{2}$ Hiroshi Tsunoda, ${ }^{1}$ Hiroyasu Inada, ${ }^{1}$ \\ Minoru Kuroki, ${ }^{2}$ Hong Sun, ${ }^{2}$ Shiro Waga, ${ }^{2}$ and Toshio Tanaka ${ }^{1}$ \\ ${ }^{1}$ Department of Molecular and Cellular Pharmacology, and \\ ${ }^{2}$ Department of Neurosurgery, Mie University School of Medicine, Mie 514-8507, Japan
}

Address correspondence to: Toshio Tanaka, Department of Molecular and Cellular Pharmacology,

Mie University School of Medicine, 2-174 Edobashi, Tsu, Mie 514-8507, Japan. Phone: 81-59-231-5006;

Fax: 81-59-232-1765; E-mail: tanaka@doc.medic.mie-u.ac.jp.

Received for publication September 28, 1998, and accepted in revised form May 20, 1999.

Delayed cerebral vasospasm after aneurysmal subarachnoid hemorrhage (SAH) causes cerebral ischemia and infarction. To date, the pathogenesis and gene expression associated with vasospasm remain poorly understood. The present study used fluorescent differential display to identify differentially expressed genes in a rat model of SAH. By using quantitative RT-PCR, we found that heme oxygenase-1 (HO-1) mRNA was prominently induced in the basilar artery and modestly in brain tissue in a rat vasospasm model. A significant correlation was observed between the degree of vasospasm and HO-1 mRNA levels in the basilar arteries exhibiting vasospasm. Intracisternal injection of antisense HO-1 oligodeoxynucleotide (ODN) significantly delayed the clearance of oxyhemoglobin and deoxyhemoglobin from the subarachnoid space and aggravated angiographic vasospasm. Antisense HO-1 ODN inhibited HO-1 induction in the basilar arteries but not in the whole brain tissue. This phenomenon was not observed in the nontreated, sense HO-1 ODN-treated, or scrambled ODN-treated arteries. We report the protective effects of HO-1 gene induction in cerebral vasospasm after SAH, a finding that should provide a novel therapeutic approach for cerebral vasospasm.

J. Clin. Invest. 104:59-66 (1999).

\section{Introduction}

Subarachnoid hemorrhage (SAH) is a common and often devastating occurrence; each year approximately 7-20 of every 100,000 people experience nontraumatic $\mathrm{SAH}(1-3)$. Despite considerable advances in diagnostic, surgical, and anesthetic techniques, and advances in perioperative management, the outcome for patients with SAH remains poor, with an overall mortality rate of $25 \%$ and significant morbidity among approximately $50 \%$ of survivors (1-3). Cerebral vasospasm, which is a major cause of morbidity and mortality after SAH (1-3), is the delayed narrowing of large-capacitance arteries at the base of the brain. Angiographic vasospasm typically develops 3-5 days after $\mathrm{SAH}$ and is most severe 7-9 days after SAH (1-3). In approximately half of all cases, a delayed neurologic ischemic deficit occurs, and despite maximal therapy, $15-20 \%$ of such patients suffer stroke or die from vasospasm (1-3). Of the patients who survive, vasospasm spontaneously resolves over the course of several days and usually disappears by the end of the second or fourth week (1-3). Recent evidence suggests that hemoglobin-induced (Hb-induced) oxidative stress plays a central role in the pathogenesis of vasospasm after SAH (4). However, the mechanism by which delayed cerebral vasospasm spontaneously resolves has not been sufficiently investigated. Clarification of the intrinsic mechanism of spasmolysis may lead to a novel therapeutic approach. In the present study, we used an improved differential display technique, fluorescent differential display (FDD) (5), to identify differentially expressed genes and to evaluate the functional significance of such genes in the basilar artery with vasospasm.

\section{Methods}

All protocols were evaluated and approved by the Animal Ethics Review Committee of the Mie University School of Medicine. Treatment of animals was in accordance with Guidelines for Animal Experiments at the Mie University School of Medicine.

Experimental model of $\mathrm{SAH}$ and study protocol. Male Sprague-Dawley rats (age 10-12 weeks, 350-450 g; Japan SLC Inc., Hamamatsu, Japan) were anesthetized by an intraperitoneal injection of chloral hydrate (400 $\mathrm{mg} / \mathrm{kg}$ ), intubated with a 16 - or 18-gauge elastic catheter, and allowed to breathe spontaneously. Rectal temperature was kept at approximately $37^{\circ} \mathrm{C}$ with an electric heating pad, and end-tidal $\mathrm{CO}_{2}$ was monitored (POET 601; Criticare System Inc., Tokyo, Japan). Anesthesia was maintained by repeated injections of chloral hydrate as needed. After cannulation of either the left or both radial arteries with 24-gauge catheters, catheters were connected with a 3-way stopcock to a pressure transducer (MK-12030-US; Baxter Healthcare Corp., Deerfield, Illinois, USA) to measure blood pressure. After angiography, rats were placed in a head 
holder with a head flexion angle of $30^{\circ}$. A midline skin incision was made from the middle of the calvarium to the lower cervical spine. The occipital bone was cleared of muscular attachments by a sharp dissection. The atlanto-occipital membrane was dissected under a surgical microscope, and a 27-gauge needle was inserted through the arachnoid membrane and into the cisterna magna. Infusions were performed over a 10 -minute period, after which the needle was withdrawn and plugged with an absorbable sponge and the wound was sutured. Throughout the course of the observation, the rats were allowed access to food and water ad libitum.

To study the time course of angiographic vasospasm, the rats were assigned to 1 of 4 groups. The 1-hemorrhage $(n=5)$ and 1 -saline $(n=5)$ groups received $0.3 \mathrm{~mL}$ of either fresh whole autologous arterial blood or saline. In the 2 -hemorrhage $(n=19)$ and 2 -saline $(n=3)$ groups, the rats were reanesthetized at 48 hours after the initial injection and then given a second injection of either blood or saline. Vertebral angiography was performed at the following time points: day 0 (before injection and at 10 minutes, 1 hour, and 3 hours after injection), day 1 , day 2 (before injection and at 10 minutes, 1 hour, and 3 hours after the injection), and days 3-14.

Differential display analyses were performed on the basilar arteries obtained from the rats at the following time points: before injection $(n=6)$ and at 10 minutes after the injection on day $0(n=6)$, day $1(n=6)$, day 7 $(n=6)$, and day $12(n=5)$ in the 2-hemorrhage group; and on day 7 in the 1-hemorrhage $(n=5), 1$-saline $(n=5)$, and 2-saline $(n=5)$ groups. Selected gene expression on differential display gel images was confirmed using quantitative RT-PCR analyses of samples obtained at identical time points $(n=3)$

Antisense heme oxygenase-1 (HO-1) oligodeoxynucleotide (ODN), sense HO-1 ODN, or scrambled ODN treatment was performed on the 1-hemorrhage rats. The efficacy of antisense ODN uptake by the basilar artery and the whole brain tissue, including the neuronal tissue and vessels, was quantified by measuring the levels of targeted HO-1 mRNA and protein in the basilar artery and the whole brain tissue (6). Vertebral angiography in antisense HO-1 ODN, sense HO-1 ODN, and scrambled ODN treatment groups ( $n=10,8$, and 8 , respectively) and quantitative RT-PCR analyses ( $n=3$ in each group) were performed before the injection of each ODN and after the injections on days 1-7. Western blot analyses were performed on the basilar arteries $(n=8$ in each group) and the whole brain tissue ( $n=3$ in each group) on day 1. To determine the effect of antisense HO-1 ODN treatment on the rate of clearance of heme arising from the injected blood, the cisternal levels of $\mathrm{Hb}$ were measured before and after the injection of each ODN from days 1-7 ( $n=3$ in each group). The rats in the 1saline group also received antisense HO-1 ODN and subsequently underwent angiography ( $n=2$ in each group).

Vertebral angiography. Rats were anesthetized, and all physiological parameters were monitored. As described previously (7), vertebral angiography was performed by a simultaneous injection of contrast medium $(0.3 \mathrm{~mL}$ of Iomeron 400; Eisai, Corp., Tokyo, Japan) into the bilateral radial arteries, or $0.6 \mathrm{~mL}$ into either the left or right radial artery. Basilar artery diameter was determined at 3 locations: $0.5 \mathrm{~mm}$ above the junction of the vertebral arteries, at the midpoint of the basilar artery, and just below the bifurcation of the posterior cerebral arteries. During angiography, exposure factors were held constant, and a radiopaque control standard was used for correction to constant magnification. Three experienced technicians unaware of the treatment groups measured the diameter of the basilar artery 9 times with a calibrated optical micrometer (Scale Lupe no. 1983; PEAK, Corp., Tokyo, Japan). NIH image and Adobe Photoshop 4.0J (Adobe Systems Tokyo, Japan) software, a high-resolution scanner (GT-9500; Epson Inc., Tokyo, Japan), and a Macintosh computer (Apple Japan Inc., Tokyo, Japan) were used to determine the mean values.

Sample collection and sacrificing of rats. At each sampling point, the rats were sacrificed with an intraperitoneal injection of chloral hydrate $(600 \mathrm{mg} / \mathrm{kg})$. The brains were rapidly removed. The basilar arteries, ventral brain stems, cerebellar hemispheres, and frontal, parietal, and occipital cortices were excised and immediately frozen in liquid nitrogen. In other rats, the whole brain was rapidly removed and then immediately frozen in liquid nitrogen. The extraction of total RNA from the specimen and reverse transcription of total RNA into first-strand cDNAs were performed on the day the specimen was harvested. The obtained cDNA solution was stored at $-30^{\circ}$ until analysis.

Cerebrospinal fluid was collected by direct puncture from the cisterna magna, as already described here, and then stored in liquid nitrogen. The rats were sacrificed, and the whole brain and the basilar arteries were stored in liquid nitrogen until the Western blot analyses.

$F D D$. FDD was performed essentially as described (5). Quantitative RT-PCR. Total RNA was extracted from the basilar arteries and brains by a modified single-step

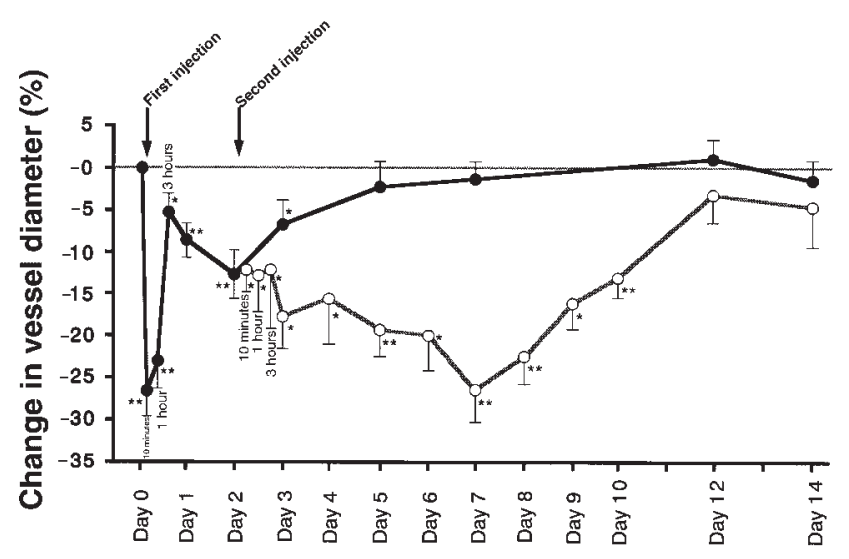

Figure 1

The percent change from baseline in the diameter of the basilar artery in the 1-hemorrhage (filled circles) and 2-hemorrhage (open circles) groups. Significantly different from the baseline values on day $0\left({ }^{*} P\right.$ $\left.<0.05,{ }^{*} P<0.01\right)$. 
acid-guanidine thiocyanate-phenol-chloroform method (8) using ISOGEN reagent (Nippon Gene, Tokyo, Japan). The ethanol-precipitated RNA fraction $(1 \mu \mathrm{g})$ was reverse transcribed using SuperScript II reverse transcriptase (GIBCO BRL, Gaithersburg, Maryland, USA), according to the manufacturer's protocol, and 50 $\mu \mathrm{L}$ of first-strand cDNA solution was obtained. Two methods were used to determine the changes in HO-1 mRNA levels: competitive RT-PCR analysis of HO- 1 and HO-2 mRNA (9), and a newly developed real-time RTPCR analysis $(10,11)$. The latter method was used because quantitation of mRNA by RT-PCR has been reported to be potentially unreliable (10).

Competitive RT-PCR analysis of HO-1 and HO-2 mRNA was performed on a DNA Thermal Cycler (PJ2000; Perkin-Elmer, Corp., Norwalk, Connecticut, USA) using reagents from the same source. Primers 5'-CAC-CAG-CCA-CAC-AGC-ACT-AC-3' and 5'-CACCCA-CCC-CTC-AAA-AGA-CA-3' were de-signed to amplify the 323- to 1,365 -bp region of the rat $\mathrm{HO}-1$ cDNA. Primers 5'-AGG-GCA-GCA-CAA-ACA-ACT-

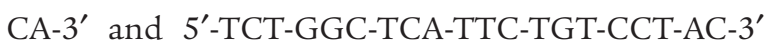
were designed to amplify the 21- to 483-bp region of the rat HO- 2 cDNA. A reaction mixture $(10 \mu \mathrm{L})$ containing $\mathrm{CDNA}$ preparation (corresponding to $10 \mathrm{ng}$ of total RNA), primers ( $0.5 \mu \mathrm{M}$ each), $1 \times$ PCR buffer (containing $1.5 \mathrm{mM} \mathrm{MgCl} 2), 125 \mu \mathrm{M}$ dNTPs, and $0.05 \mathrm{U} / \mu \mathrm{L}$ AmpliTaq Gold (Perkin-Elmer Corp.) was used. Samples were kept at $94^{\circ} \mathrm{C}$ for 15 minutes and then subjected to thermocycling ( 28 cycles of 1 minute at $94^{\circ}$, 1 minute at $57^{\circ}$, and 1 minute at $72^{\circ}$, with a final extension at $72^{\circ}$ for 5 minutes). Primer sequences for rat $\beta$-actin (internal controls) were $5^{\prime}$-GGG-AAA-TCGTGC-GTG-ACA-T- $3^{\prime}$ and $5^{\prime}$-CAG-GAG-GAG-CAATGA-TCT-T- $3^{\prime}$. To amplify $\beta$-actin cDNA, samples were kept at $94^{\circ}$ for 15 minutes and subjected to thermocycling $\left(20\right.$ cycles of 30 seconds at $94^{\circ}, 30$ seconds at $50^{\circ}$, and 2 minutes at $72^{\circ}$, with a final extension at $72^{\circ}$ for 5 minutes). Aliquots ( $3 \mu \mathrm{L}$ ) in gel loading buffer (final concentrations of $25 \mathrm{mg} / \mathrm{mL}$ blue dextran and $6 \%$ glycerol) were loaded and electrophoretically separated on $2 \%$ agarose gels. After staining in $0.5 \mu \mathrm{g} / \mathrm{mL}$ ethidium bromide solution for 30 minutes and rinsing with fresh distilled deionized water, each gel was scanned using a fluorescent image analyzer (FMBIO II; Hitachi, Yokohama, Japan). The density of each band was determined on a Macintosh computer (Apple Japan Inc.) with FMBIO Analysis 6.0 software (Hitachi).

For real-time RT-PCR analysis, cDNA corresponding to $10 \mathrm{ng}$ of total RNA was added to a $50-\mu \mathrm{L}$ reaction mixture. The reaction master mix was prepared according to the manufacturer's protocol to yield final concentrations of $1 \times$ TaqMan buffer A, $3.5 \mathrm{mM} \mathrm{MgCl}_{2}, 30$ $\mathrm{nM}$ primers, $20 \mathrm{nM}$ probe, $0.2 \mathrm{mM}$ dATP, $0.2 \mathrm{mM}$ dCTP, $0.2 \mathrm{mM}$ dGTP, $0.4 \mathrm{mM}$ dUTP, $0.01 \mathrm{U} / \mu \mathrm{L}$ AmpErase uracil $N$-glycosylase, and $0.025 \mathrm{U} / \mu \mathrm{L}$ AmpliTaq Gold. The primers were provided by Oligo Service (Tsukuba, Japan), and the probes by Perkin-Elmer Japan (Urayasu, Japan). The primers used in this study

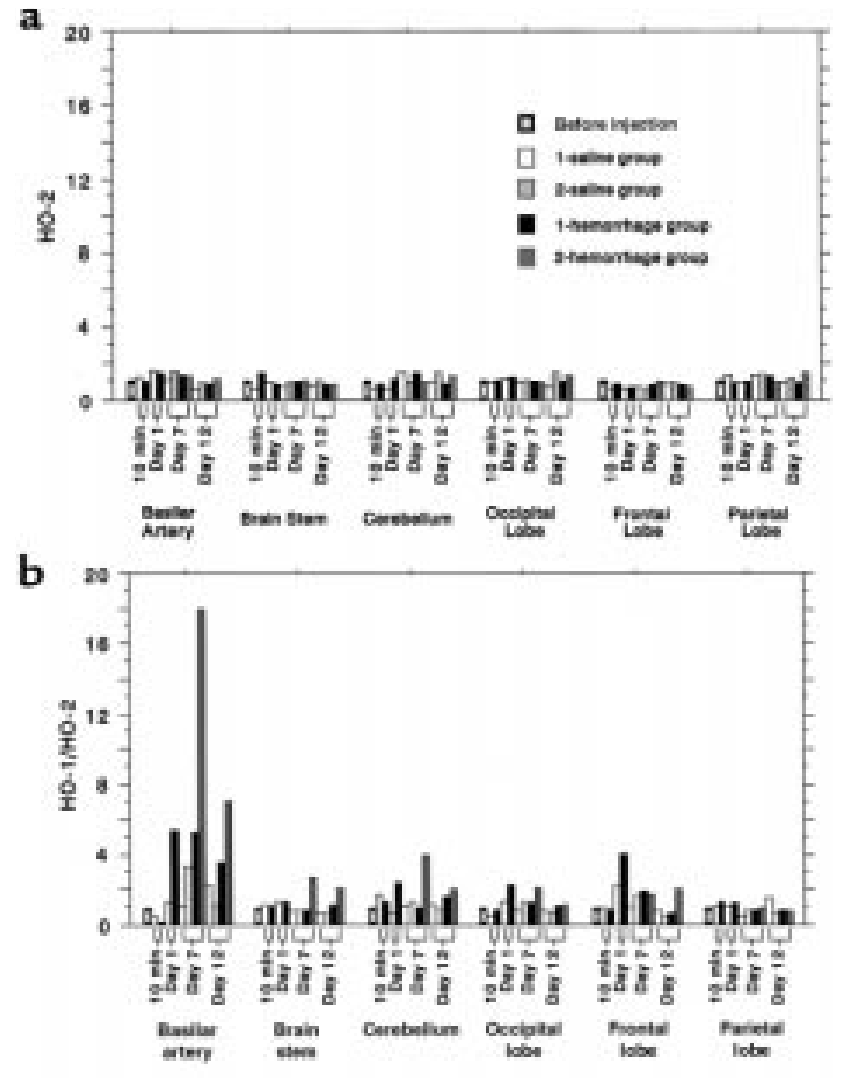

Figure 2

(a) HO-2 mRNA levels, which did not change appreciably after the injection of either blood or saline, were determined using real-time RT-PCR analysis. Data presented are means of duplicate determinations $(n=3)$, and the variance of data was less than $10 \%$. (b) The HO-1 mRNA levels in the same samples as a were determined using real-time RT-PCR analysis and were expressed as a ratio of HO-1 mRNA to HO-2 mRNA. Data presented are means of duplicate determinations $(n=3)$, and the variance of data was less than $10 \%$. Note that the expression level of HO-1 mRNA in the basilar artery was the most prominent among all examined regions of the brain.

were $5^{\prime}$-CGA-CAG-CAT-GTC-CCA-GGA-TT-3' and $5^{\prime}$-TCG-CTC-TAT-CTC-CTC-TTC-CAG-G-3' for rat HO-1; 5'-TTC-TAC-CTG-TTC-GAG-CAT-GTG-G-3' and $5^{\prime}$-TGT-TAG-CAT-GGA-GCC-AGC-CT-3' for rat HO-2; and 5'-TGA-ACA-CGG-CAT TGT-AAC-CAA-C$3^{\prime}$ and 5'-CAG-TGG-TAC-GAC-CAG-AGG-CAT-A-3' for rat $\beta$-actin. The probes were 5' (FAM)-TAA-GCTGGT-GAT-GGC-CTC-CTT-GTA-CC-(TAMRA)p3' for rat HO-1; 5' (FAM)-TTC-TAC-CGC-GCC-AGG-ATGAAT-G-(TAMRA)p3' for rat HO-2; and 5' (FAM)-TGTA C G-TAG-C CA - T C C - A G G - C T G - T G T-T G(TAMRA)p3' for rat $\beta$-actin. PCR reactions and the resulting relative increase in reporter fluorescent dye emission were monitored in real time using a 7700 sequence detector (Perkin-Elmer, Corp.) Signals were analyzed using the sequence detector 1.0 program (Perkin-Elmer). Conditions were as follows: 1 cycle at $50^{\circ}$ for 2 minutes, 1 cycle at $95^{\circ}$ for 10 minutes, and 40 cycles at $95^{\circ}$ for 15 seconds and $60^{\circ}$ for 1 minute. The standard curves for HO-1, HO-2, and $\beta$-actin were gen- 


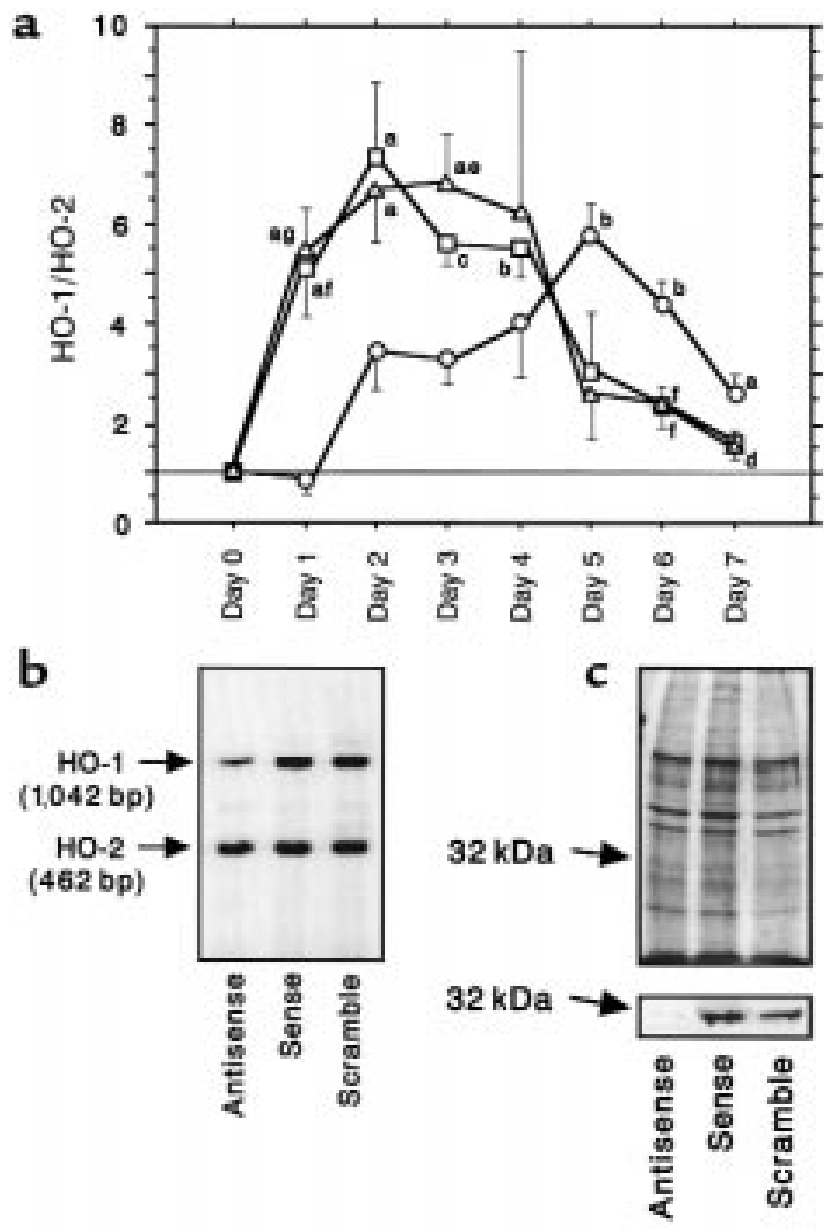

Figure 3

(a) In the 1-hemorrhage rats, the HO-1 mRNA levels in the basilar arteries after the treatment with antisense HO-1 ODN (circles, $n=$ 3 ), sense HO-1 ODN (squares, $n=3$ ), or scrambled ODN (triangles, $n=3$ ) were determined using real-time RT-PCR analysis and were expressed as a ratio of HO-1 mRNA to HO-2 mRNA. Significantly different from the baseline values on day 0 ( ${ }^{a} P<0.05$, ${ }^{b} P<0.025$, ${ }^{c} P$ $<0.01$ ). Significantly different from the values for the antisense $\mathrm{HO}-$ 1 ODN treatment ( ${ }^{\mathrm{d}} P<0.05$, $\left.{ }^{\mathrm{e}} P<0.025,{ }^{\mathrm{f}} P<0.01, \mathrm{~g} P<0.005\right)$. (b) Competitive RT-PCR analysis of HO-1 and HO-2 mRNA in the basilar arteries on day 1 after the injection of blood. Antisense $\mathrm{HO}-1$ ODN prevented the induction of HO-1 mRNA but did not affect $\mathrm{HO}-$ 2 mRNA levels. (c) The basilar arteries on day 1 after the injection of blood were also analyzed by electrophoresis on a $12.5 \%$ SDS-polyacrylamide gel, followed by Coomassie staining (top) or Western blotting with anti-HO-1 antibodies (bottom). Antisense HO-1 ODN prevented the induction of $\mathrm{HO}-1$ protein.

erated by serial dilution of cDNA obtained from the basilar arteries harvested from the rats on day 7 in the 2-hemorrhage group, and from the frontal cortices and basilar arteries of normal rats.

Intrathecal injection of ODN. Phosphorothioate ODNs were provided by Oligo Service. The sequences of ODN against rat $\mathrm{HO}-1$ used in this study were antisense: 5'-ACA-CCT-GAC-CCT-TCT-3'; sense: 5'-AGA-AGGGTC-AGG-TGT-3' (+113 to +127 of rat sequence); and scrambled: 5'-CGT-ACT-CTC-ACT-CAC-3' - which comprised all the base pairs of the antisense codon in random order. The sequence homologies were checked against the reported DNA sequences present in the BLASTN program. The antisense, sense, and scrambled ODNs were each diluted in saline to a concentration of $400 \mu \mathrm{M}$. The ODN solution was injected twice into the cisterna magna; the first injection $(0.35 \mathrm{~mL})$ was administered at 24 hours before the injection of either blood or saline, and the second injection $(0.05 \mathrm{~mL})$ was administered simultaneously with the injection of either blood or saline on day 0 .

Western blot analyses. For HO-1 immunoblots, frozen tissues were homogenized in boiling lysis buffer (1\% SDS, $1 \mathrm{mM} \mathrm{Na}_{3} \mathrm{VO}_{4}, 10 \mathrm{mM}$ Tris [pH 7.4]) containing protease inhibitors $(1 \mu \mathrm{M}$ APMSF, $5 \mathrm{mg} / \mathrm{L}$ trypsin inhibitor, $5 \mathrm{mg}$ /L leupeptin). The protein concentration of the lysates was determined by Coomassie Blue dyebinding assay (Bio-Rad Laboratories Inc., Hercules, California, USA). An equal volume of $2 \times \mathrm{SDS} /$ sample buffer (0.1 M Tris-HCl [pH 6.8], 4 \% SDS, 12\% 2-mercaptoethanol) was added, and the samples were boiled for 3 minutes. Samples $(10 \mu \mathrm{g})$ were subjected to electrophoresis in a $12.5 \%$ SDS-polyacrylamide gel and then transferred electrophoretically onto a PVDF membrane. The membranes were incubated for 1 hour with mouse $\mathrm{mAb}$ against rat HO-1 (1:300 dilution; StressGen Biotechnologies Corp., Victoria, British Columbia, Canada), followed by incubation with sheep anti-mouse IgG antibody (1: 2,500 dilution; Amersham Corp., Burlington, Massachusetts, USA) for 30 minutes. Signal development was carried out using an ECL detection kit (Amersham Corp.).

Measurements of $\mathrm{Hb}$. The concentrations of oxyhemoglobin $(\mathrm{OxyHb})$, deoxyhemoglobin (DeoxyHb), and methemoglobin (MetHb) in the cerebrospinal fluid were measured by spectrophotometry as described by FoghAndersen et al. (12). The absorbance of the samples was measured 3 times at 6 different wavelengths $(535,560$, $577,622,636$, and $670 \AA$ ) , and the mean absorbance value was used to calculate the $\mathrm{Hb}$ concentrations.

Statistical analysis. All data were expressed as mean \pm SEM. Comparisons within groups were made by paired or unpaired $t$ tests, and intergroup comparisons among the 3 groups were determined by 1-way ANOVA. $P<0.05$ was considered statistically significant.

\section{Results}

SAH induces angiographic vasospasm of the basilar artery. Rats were assigned to 1 of 4 groups: 1-hemorrhage, 2hemorrhage, 1 -saline, and 2 -saline injected groups. The 1 - and 2-hemorrhage rats were given 1 and 2 injections (48 hours apart), respectively, of autologous blood into the cisterna magna. The saline groups were given saline injections according to the same protocol. No rats were observed to develop neurologic deficits. Comparisons of mean values for body weight, mean arterial blood pressure, heart rate, and end-tidal $\mathrm{CO}_{2}$ revealed no significant differences among the groups (data not shown). The 1- and 2-hemorrhage rats showed biphasic vasospasms occurring at 10 minutes (early 


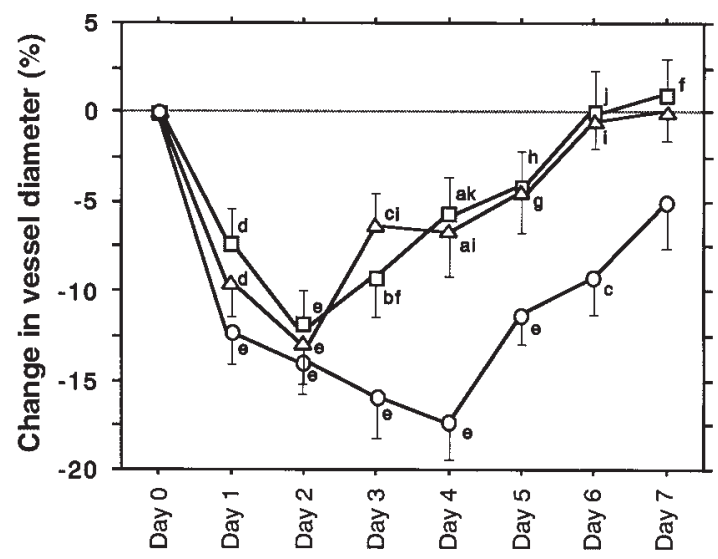

Figure 4

The percent change from baseline of the basilar artery diameter in the 1-hemorrhage rats after the treatment with antisense HO-1 ODN (circles, $n=10$ ), sense HO-1 ODN (squares, $n=8$ ), or scrambled ODN (triangles, $n=8$ ). Significantly different from the baseline values on day 0 ( ${ }^{\mathrm{a}} P<0.01,{ }^{\mathrm{b}} P<0.001,{ }^{\mathrm{c}} P<0.0005,{ }^{\mathrm{d}} P<0.00025,{ }^{\mathrm{e}} P<$ $0.0001)$. Significantly different from the values for the antisense $\mathrm{HO}$ 1 ODN treatment $\left({ }^{f} P<0.05, \mathrm{~g} P<0.025, \mathrm{~h} P<0.01,{ }^{\mathrm{i}} P<0.005, \mathrm{j} P<\right.$ $0.0025, \mathrm{k} P<0.0005)$.

vasospasm) and on days 2 and 7 (delayed vasospasm) after blood injections (Figure 1). Delayed vasospasm in the 2-hemorrhage rats resembled human vasospasm. No significant vasospasm was observed in the saline groups (data not shown).

SAH induces HO-1 $m R N A$ in the basilar artery and brain. FDD was performed on the basilar arteries with no, early, and delayed vasospasms. From FDD fingerprints, we identified changes in intensity in approximately $3 \%$ (280 bands) of the 9,642 bands specific for delayed vasospasm in the 2 -hemorrhage rats. We focused on 1 upregulated cDNA fragment, rat HO-1 gene, which coincided with the development of delayed vasospasm.

HO-1 mRNA levels in the basilar artery and brain obtained at identical time points were determined by 2 quantitative RT-PCR analysis methods. HO-1 mRNA levels were expressed as a ratio of the HO-2 mRNA level for normalization, given that the mRNA levels of HO2 and $\beta$-actin in the basilar artery and brain after an injection of either blood or saline were unchanged (Figure 2a). Real-time RT-PCR analysis demonstrated that HO-1 mRNA was exclusively induced in the basilar artery and brain in SAH groups, but not in the saline groups (Figure 2b). Higher levels of HO-1 mRNA expression were observed in the 2 -hemorrhage rats, in which a comparatively larger amount of blood was observed than in the 1-hemorrhage rats. Interestingly, among all the tissues evaluated, the most prominent levels of HO-1 mRNA expression were observed in the basilar artery (Figure $2 b$ ). In spite of an identical distribution of SAH, the levels of HO-1 mRNA in the basilar artery with delayed vasospasm were approximately 7 times higher than in the ventral brain stem on day 7 in the 2-hemorrhage group (Figure 2b). A significant linear correlation was observed between the degree of delayed vasospasm and HO-1 mRNA levels in the basilar artery, ventral brain stem, and cerebellum $(r=0.949$, $P<0.0025 ; r=0.829, P<0.05$; and $r=0.949, P<0.0025$, respectively). HO-1 mRNA was not induced in the basilar artery with early vasospasm or in the brain $10 \mathrm{~min}$ utes after the injection of blood on day 0 (Figure $2 \mathrm{~b}$ ). Competitive RT-PCR analysis of $\mathrm{HO}-1$ and $\mathrm{HO}-2$ mRNA yielded similar results (data not shown).

Effect of antisense HO-1 ODN treatment on delayed vasospasm. To determine the functional significance of HO-1 gene induction in the basilar artery and brain, we examined the effects of selective $\mathrm{HO}-1$ inhibition using antisense $\mathrm{HO}-1$ ODN on angiographic vasospasm. Antisense HO-1 ODN, sense HO-1 ODN, or scrambled ODN treatment was performed on 1-hemorrhage rats. In the basilar artery, a successful blockage of HO-1 mRNA upregulation was observed after 2 intrathecal injections of antisense HO-1 ODN (Figure 3 , $a$ and $b$ ). The antisense HO-1 ODN also prevented the upregulation of HO-1 protein in the basilar artery (Figure $3 \mathrm{c}$ ). The levels of HO-2 and $\beta$-actin mRNA were unaffected by antisense HO-1 ODN, sense HO-1 ODN, or scrambled ODN treatment (Figure 3b). Sense HO-1 ODN or scrambled ODN treatment did not affect HO-1 gene expression levels nor delayed vasospasm in the basilar artery (Figures 3a and 4). On the other hand, antisense $\mathrm{HO}-1$ ODN significantly inhibited $\mathrm{HO}-1$ induction in the basilar artery until day 4 after SAH (Figure 3a). Moreover, antisense HO-1 ODN significantly aggravated vasospasm (Figure 4). The peak time of vasospasm was delayed from day 2 to day 4 (Figure 4). Vasospasm was present up until day 7 , at which point it had already resolved in the control rats (Figure 4). It is noteworthy that HO-1 mRNA in the basilar artery was significantly induced from day 5 to day 7 after the antisense ODN treatment, when vasospasm was significantly attenuated (Figures $3 \mathrm{a}$ and 4 ). These results strongly suggest that HO-1 gene induction in the basilar artery has antivasospastic effects. The antisense HO-1 ODN treatment did not affect the diameter of basilar artery in the saline group, suggesting that antisense HO-1 ODN does not itself exacerbate vasospasm (data not shown).

HO-1 mRNA was significantly induced on day 1 in the whole brain tissue, including the neuronal tissue and vessels (Figure 5a). The magnitude of $\mathrm{HO}-1$ induction in the whole brain tissue was much smaller than in the basilar artery (Figures 3a and 5a). The HO-1 responses in the whole brain tissue after the antisense HO-1 ODN treatment were not significantly different from those in the control groups (Figure 5, a and b). These results suggest that HO-1 levels in the whole brain tissue may reflect $\mathrm{HO}-1$ induction in the neuronal tissue, which was not affected by antisense HO-1 ODN. HO-1 induction has been observed in neuronal tissue, specifically in glial cells after SAH $(7,13-15)$. The inhibitory effects of antisense HO-1 ODN treatment on HO-1 induction in the basilar artery are likely masked by the larger amounts of $\mathrm{HO}-1$ induction in glial cells. Thus, intrathecal injections of antisense HO- 


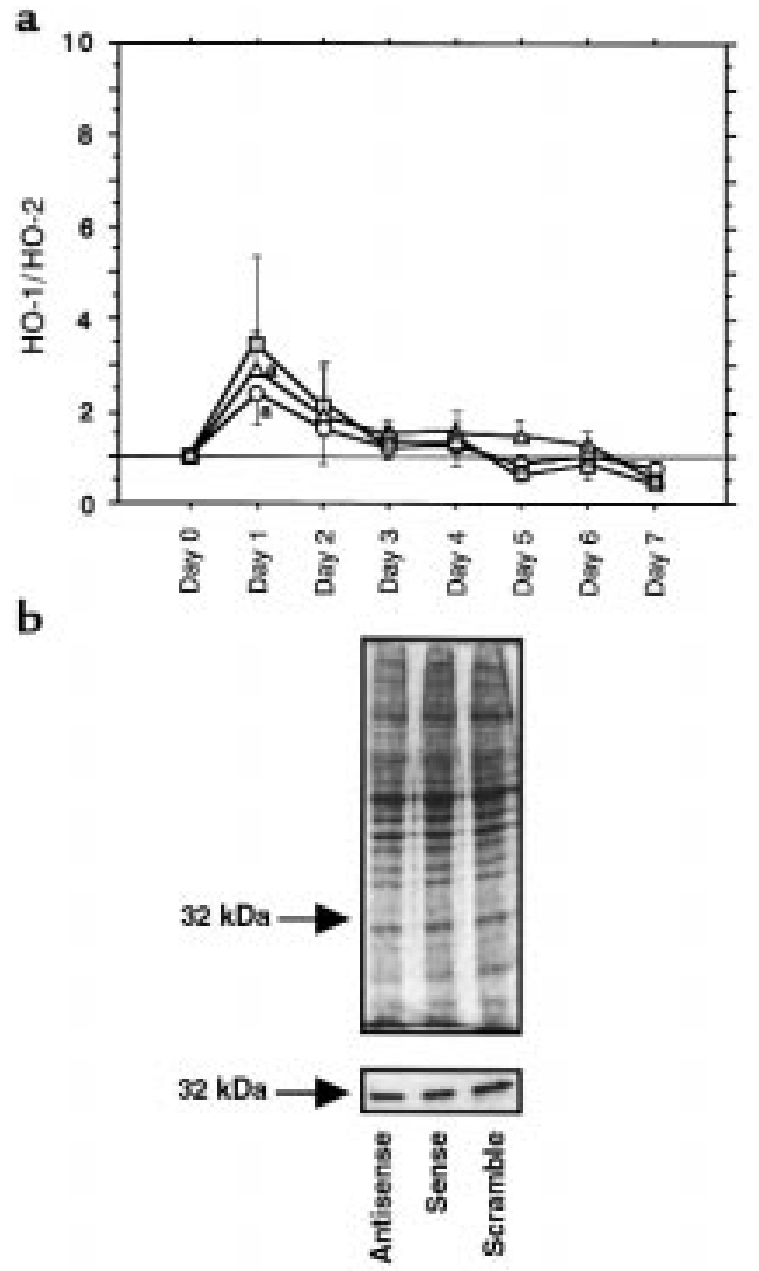

Figure 5

(a) HO-1 mRNA levels in the whole brain tissue, including neuronal tissue and vessels, in the 1-hemorrhage rats after the treatment with antisense HO- 1 ODN (circles, $n=3$ ), sense HO- 1 ODN (squares, $n$ $=3$ ), or scrambled ODN (triangles, $n=3$ ). Significantly different from the baseline values on day $0\left({ }^{a} P<0.05\right)$. No significant differences were observed among the 3 groups. (b) The whole brain tissue on day 1 after the injection of blood was analyzed by electrophoresis on a $12.5 \%$ SDS-polyacrylamide gel, followed by Coomassie staining (top) or Western blotting with anti-HO-1 antibodies (bottom). No significant differences were observed among the 3 groups.

$1 \mathrm{ODN}$ affected the HO-1 levels in the basilar artery but not in the neuronal tissue.

Effect of antisense HO-1 ODN treatment on clearance of $\mathrm{Hb}$ from the subarachnoid space. Because our method of measuring $\mathrm{Hb}$ levels did not allow us to continue to use anaerobic conditions, we had to estimate the sum of $\mathrm{OxyHb}$ and DeoxyHb, and MetHb levels. The cisternal levels of OxyHb and DeoxyHb in the control groups peaked on day 2 , when delayed vasospasm was most prominent, and fell sharply on day 3 (Figure 6a). In contrast, the levels of $\mathrm{OxyHb}$ and DeoxyHb after the antisense HO-1 ODN treatment were higher on day 3 than day 2 , and began to fall on day 4 (Figure 6a). The levels of OxyHb and DeoxyHb after antisense HO-1 ODN treatment were significantly higher than in the control groups on days 4 and 5 (Figure 6a). In contrast to $\mathrm{OxyHb}$ and DeoxyHb, the levels of MetHb did not change appreciably in the control groups (Figure 6b). The levels of Met $\mathrm{Hb}$ were significantly higher on day 5 after antisense HO-1 ODN treatment than at baseline, but they did not differ significantly from those of the control groups (Figure 6b). Thus, aggravation of delayed vasospasm after antisense HO-1 ODN treatment may be due to the delayed clearance of $\mathrm{OxyHb}$ and DeoxyHb in the subarachnoid space.

\section{Discussion}

Recent studies support the hypothesis that HO-1 induction plays an important role in cellular protection against both heme- and non-heme-mediated oxidative injury (16). The magnitude of HO-1 induction after oxidative stress, the wide distribution of this enzyme in systemic tissues, and the intriguing biologic activity of its catalytic by-products make HO- 1 a highly attractive candidate for maintaining cellular homeostasis in response to oxidative stress (16-21). The present study demonstrated upregulation of HO-1 mRNA in the cerebral arteries and, more importantly, the augmentation and prolongation of delayed vasospasm by antisense HO-1 ODN treatment after SAH.

$\mathrm{HO}$ is the rate-limiting enzyme in the heme-degradative pathway (22). All isozymes of HO, HO-1 (an inducible isozyme), HO-2 (a noninducible isozyme), and HO-3 (a recently identified isozyme) metabolize heme in $\mathrm{Hb}$ and generate carbon monoxide (CO), free iron (ferric iron), and biliverdin (subsequently reduced to bilirubin) $(22,23)$. Hb is a potentially toxic molecule (24). All available evidence suggests that the initial reaction that leads to the formation of all potentially toxic compounds is the (auto)oxidation of $\mathrm{OxyHb}$ and $\mathrm{DeoxyHb}$ to MetHb (24). A major factor that mediates delayed vasospasm has also been reported to be OxyHb and DeoxyHb, but not MetHb $(4,25)$. The induction of $\mathrm{HO}$ can have dual beneficial effects against the toxicities of $\mathrm{Hb}$ : it removes $\mathrm{Hb}$ itself and also synthesizes the antioxidant bilirubin (24). Although the catalysis of heme by $\mathrm{HO}$ also releases pro-oxidant free iron, free iron has less pro-oxidant activity than intact $\mathrm{Hb}$ (24). Furthermore, this released iron may induce ferritin, and iron sequestration by ferritin may be the underlying mechanism responsible for the protective effects of Hb-induced HO-1 (16).

Subarachnoid blood is largely resolved within 48 hours in the rat (26). Reportedly, blood is cleared from the subarachnoid spaces through the following routes: (a) erythrocytes and $\mathrm{Hb}$ are removed via the arachnoid villi $(27,28)$; (b) erythrocytes undergo phagocytosis by cells of the pial membrane and free subarachnoid macrophages (26); and (c) brain glial cells may play a role in metabolizing extracellular heme (15). HO-1 induction may be necessary for the metabolism of extracellular heme in cells of the pial membrane, free subarachnoid macrophages, and brain glial cells (15). Thus, one explanation for the enhanced vasospasm 
observed in the present study may be a delayed clearance of SAH by HO-1 inhibition. Indeed, the cisternal levels of $\mathrm{OxyHb}$ and DeoxyHb in the antisense HO-1 ODN group were significantly higher than in other groups up until day 5. OxyHb and DeoxyHb have been reported to be responsible for evoking delayed vasospasm $(4,25)$.

Several studies have demonstrated HO-1 induction in glial cells throughout the brain after SAH (7, 13-15). Turner et al. (15) suggested that HO-1 induction in glia may be in response to the uptake of heme into the glia and heme metabolism by the glia. Glial metabolism of $\mathrm{Hb}$ is believed to decrease extracellular $\mathrm{Hb}$ and to decrease vasospasm due to extracellular $\mathrm{Hb}(15)$. In the present study, HO-1 mRNA was also induced in the basilar artery after SAH. Antisense HO-1 ODN significantly delayed the clearance of extracellular $\mathrm{Hb}$ and also enhanced delayed vasospasm. However, antisense HO-1 ODN inhibited the HO- 1 induction exclusively in the cerebral arteries, and not in other regions of the brain. Thus, the delayed clearance of $\mathrm{Hb}$ after antisense $\mathrm{HO}-1$ ODN treatment might be attributable to the decreased heme metabolism in the vascular cells surrounding the brain surface.

In vascular cells, HO-1 expression has been observed in the endothelial and smooth muscle cells under certain pathophysiological conditions (16, 20, 21, 29, 30). As a by-product of $\mathrm{HO}, \mathrm{CO}$ is a gas molecule that shares some of the properties of nitric oxide, inasmuch as it binds to the heme moiety of cytosolic guanylyl cyclase to produce cGMP $(21,31)$. HO-1 is induced by hypoxia in vascular smooth muscle cells; its by-product, CO, promotes the accumulation of cGMP (20). Increased cGMP causes smooth-muscle relaxation (20). Moreover, smooth muscle cell-derived CO inhibits the production of the endothelium-derived vasoactive agents endothelin-1 and PDGF-B (32).

In a rat model of $\mathrm{SAH}$, biphasic vasospasm has been observed in the basilar artery after an injection of blood (33). It is rat delayed vasospasm, not early vasospasm, that mimics human vasospasm, which causes morbidity and mortality after SAH in clinical practice $(1,2)$. A recent study has demonstrated that intracisternal administration of antisense ODN for preproendothelin-1 successfully inhibits its mRNA levels in the basilar arteries and prevents early vasospasm (34). In the present study, we demonstrated that antisense HO-1 ODN significantly aggravated delayed vasospasm by selective $\mathrm{HO}-1$ inhibition in the basilar arteries. HO-1 mRNA was significantly induced from day 5 to day 7 at the resolutional stage of delayed vasospasm after antisense ODN treatment. HO-1 mRNA levels were upregulated in delayed vasospasm and not in early vasospasm. HO-1 mRNA was also induced in other brain tissues after SAH. However, the degree of HO-1 induction and its time course in the brain did not correlate with those of delayed vasospasm. Indeed, antisense HO-1 ODN sig-
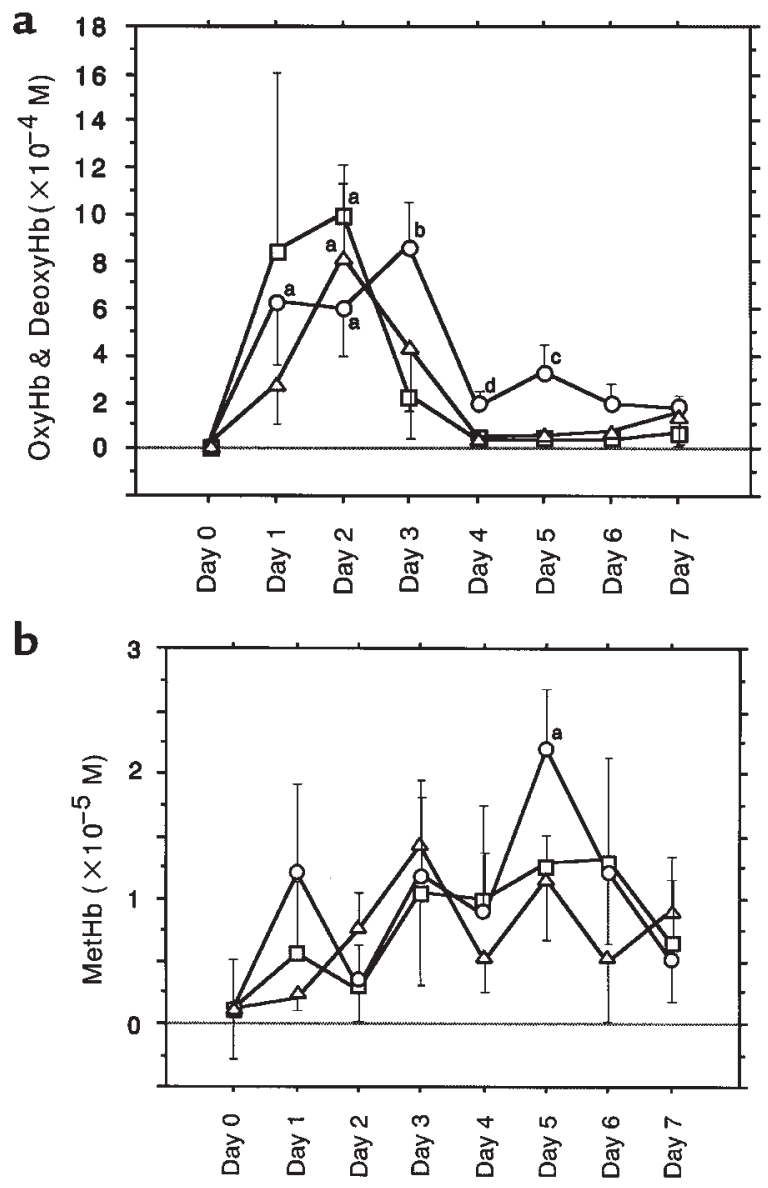

Figure 6

(a) Cisternal levels of $\mathrm{OxyHb}$ and DeoxyHb in the 1-hemorrhage rats after treatment with antisense HO-1 ODN (circles, $n=3$ ), sense HO1 ODN (squares, $n=3$ ), or scrambled ODN (triangles, $n=3$ ). Significantly different from the baseline values on day 0 ( $P<<0.025$, b $P$ $<0.001)$. Significantly different from the values for the antisense $\mathrm{HO}-$ 1 ODN treatment $\left({ }^{c} P<0.05,{ }^{d} P<0.005\right)$. (b) Cisternal levels of $\mathrm{Met} \mathrm{Hb}$ in the same samples as $\mathbf{a}$. Significantly different from the baseline values on day 0 (a $P<0.025)$. No significant differences were observed among the 3 groups.

nificantly inhibited HO-1 induction in the basilar arteries but not in the brain. Thus, HO-1 induction in the artery wall is an intrinsic regulatory mechanism that acts against delayed vasospasm. There are HO1-deficient knockout mice available that may be also suited for evaluation of this enzyme in the pathophysiology of delayed vasospasm $(35,36)$.

In conclusion, we found a remarkable level of HO-1 mRNA upregulation in the basilar artery, which may have been closely correlated with the occurrence of delayed vasospasm after SAH. In the present report, we clearly demonstrate that intrathecal administration of antisense HO-1 ODN aggravates vasospasm, a finding that suggests a spasmolytic effect for $\mathrm{HO}-1$ gene induction. Therapeutic gene induction of $\mathrm{HO}-1$ may prove to be a novel approach for the prevention and treatment of such $\mathrm{Hb}$-induced pathologic conditions as delayed cerebral vasospasm. 


\section{Acknowledgments}

This work was supported in part by grants-in-aid for Scientific Research (to T. Tanaka and K. Kanamaru) and International Scientific Research (to T. Tanaka) from the Ministry of Education, Science, Sports and Culture, and by the Grant for Pediatric Diseases from the Ministry of Health and Welfare, Japan (to T. Tanaka). This work was also supported in part by grants-in-aid (1996-1997) for the research projects on Muscle Regulation (to T. Tanaka) and Cerebral Vasospasm (to K. Kanamaru) from the Mie Medical Research Foundation.

1. Mayberg, M.R., et al. 1994. Guidelines for the management of aneurysmal subarachnoid hemorrhage. Circulation. 90:2592-2605.

2. Dorsch, N.W.C. 1995. Cerebral arterial vasospasm: a clinical review. $\mathrm{Br}$. J. Neurosurg. 9:403-412.

3. Linn, F.H.H., Rinkel, G.J.E., Algra, A., and van Gijn, J. 1996. Incidence of subarachnoid hemorrhage: role of region, year, and rate of computed tomography: a meta-analysis. Stroke. 27:625-629.

4. Macdonald, R.L., and Weir, B.K.A. 1991. A review of hemoglobin and the pathogenesis of cerebral vasospasm. Stroke. 22:971-982.

5. Ito, T., et al. 1994. Fluorescent differential display: arbitrarily primed RTPCR fingerprinting on an automated DNA sequencer. FEBS Lett. 351:231-236.

6. Wagner, R.W. 1994. Gene inhibition using antisense oligodeoxynucleotides. Nature. 372:333-335.

7. Kuroki, M., Kanamaru, K., Suzuki, H., Waga, S., and Semba, R. 1998. Effect of vasospasm on heme oxygenases in a rat model of subarachnoid hemorrhage. Stroke. 29:683-689.

8. Chomczynski, P., and Sacchi, N. 1987. Single-step method of RNA isolation by acid guanidinium thiocyanate-phenol-chloroform extraction. Anal. Biochem. 162:156-159.

9. Kutty, R.K., et al. 1994. RT-PCR assay for heme oxygenase-1 and heme oxygenase-2: a sensitive method to estimate cellular oxidative damage. Ann. NY Acad. Sci. 738:427-430.

10. Heid, C.A., Stevens, J., Livak, K.J., and Williams, P.M. 1996. Real time quantitative PCR. Genome Res. 6:986-994.

11. Gibson, U.E.M., Heid, C.A., and Williams, P.M. 1996. A novel method for real time quantitative RT-PCR. Genome Res. 6:995-1001.

12. Fogh-Andersen, N., Siggaard-Andersen, O., Lundsgaard, F.C., and Wimberley, P.D. 1987. Diode-array spectrophotometry for simultaneous measurement of hemoglobin pigments. Clin. Chim. Acta. 166:283-289.

13. Matz, P., et al. 1996. Heme-oxygenase-1 induction in glia throughout rat brain following experimental subarachnoid hemorrhage. Brain Res. 713:211-222.

14. Matz, P.G., et al. 1996. Focal hyperexpression of hemeoxygenase-1 protein and messenger RNA in rat brain caused by cellular stress following subarachnoid injections of lysed blood. J. Neurosurg. 85:892-900.

15. Turner, C.P., et al. 1998. Heme oxygenase-1 is induced in glia through out brain by subarachnoid hemoglobin. J. Cereb. Blood Flow Metab. 18:257-273.
16. Choi, A.M.K., and Alam, J. 1996. Heme oxygenase-1: function, regulation, and implication of a novel stress-inducible protein in oxidantinduced lung injury. Am. J. Respir. Cell Mol. Biol. 15:9-19.

17. Maines, M.D. 1988. Heme oxygenase: function, multiplicity, regulatory mechanism, and clinical applications. FASEB J. 2:2557-2568.

18. Goda, N., et al. 1998. Distribution of heme oxygenase isoforms in rat liver. J. Clin. Invest. 101:604-612.

19. Verma, A., Hirsch, D.J., Glatt, C.E., Ronnet, G.V., and Snyder, S.H. 1993. Carbon monoxide: a putative neural messenger. Science. 259:381-384.

20. Morita, T., Perrella, M.A., Lee, M.E., and Kourembanas, S. 1995. Smooth muscle cell-derived carbon monoxide is a regulator of vascular cGMP. Proc. Natl. Acad. Sci. USA. 92:1475-1479.

21. Maines, M.D. 1997. The heme oxygenase system: a regulator of second messenger gases. Annu. Rev. Pharmacol. Toxicol. 37:517-554.

22. Shibahara, S. 1994. Heme oxygenase-regulation of and physiological implication in heme catabolism. In Regulation of heme protein synthesis. S. Fujita, editor. AlphaMed Press. Miamiburg, OH. 103-116.

23. Mccoubrey, W.K., Jr., Huang, T.J., and Maines, M.D. 1997. Isolation and characterization of a cDNA from the rat brain that encodes hemoprotein heme oxygenase-3. Eur. J. Biochem. 247:725-732.

24. Everse, J., and Hsia, N. 1997. The toxicities of native and modified hemoglobins. Free Radic. Biol. Med. 22:1075-1099.

25. Pluta, R.M., Afshar, J.K.B., Boock, R.J., and Oldfield, E.H. 1998. Temporal changes in perivascular concentration of oxyhemoglobin, deoxyhemoglobin, and methemoglobin after subarachnoid hemorrhage. J. Neurosurg. 88:557-561.

26. Jackowski, A., Crockard, A., Burnstock, G., Russell, R.R., and Kristek, F. 1990. The time course of intracranial pathophysiological changes following experimental subarachnoid haemorrhage in the rat. J. Cereb. Blood Flow Metab. 10:835-849.

27. Simmond, W.J. 1953. Absorption of blood from cerebrospinal fluid in animals. Aust. J. Exp. Biol. Med. Sci. 30:261-270.

28. Alksne, J.F., and Lovings, E. 1972. The role of the arachnoid villi in the removal of red blood cells from the subarachnoid space. An electron microscopic study in the dog. J. Neurosurg. 36:192-200.

29. Raju, V.S., and Maines, M.D. 1996. Renal ischemia/reperfusion up-regulates heme-oxygenase-1 (HSP32) expression and increases cGMP in rat heart. J. Pharmacol. Exp. Ther. 277:1814-1822.

30. Hancock, W.W., Buelow, R., Sayegh, M.H., and Turka, L.A. 1998. Antibody-induced transplant arteriosclerosis is prevented by graft expression of anti-oxidant and anti-apoptotic genes. Nat. Med. 4:1392-1396.

31. Dawson, T.M., and Snyder, S.H. 1994. Gases as biological messengers: nitric oxide and carbon monoxide in the brain. J. Neurosci. 14:5147-5159.

32. Morita, T., and Kourembanas, S. 1995. Endothelial cell expression of vasoconstrictors and growth factors is regulated by smooth muscle cell-derived carbon monoxide. J. Clin. Invest. 96:2676-2682.

33. Delgado, T.J., Brismar, J., and Svendgaard, N.A. 1985. Subarachnoid haemorrhage in the rat: angiography and fluorescence microscopy of the major cerebral arteries. Stroke. 16:595-602.

34. Onoda, K., et al. 1996. Inhibition of vascular contraction by intracisternal administration of preproendothelin-1 mRNA antisense oligo DNA in a rat experimental vasospasm model. J. Neurosurg. 85:846-852.

35. Poss, K.D., and Tonegawa, S. 1997. Heme oxygenase 1 is required for mammalian iron reutilization. Proc. Natl. Acad. Sci. USA. 94:10919-10924.

36. Poss, K.D., and Tonegawa, S. 1997. Reduced stress defense in heme oxygenase 1-deficient cells. Proc. Natl. Acad. Sci. USA. 94:10925-10930. 